\title{
Long-time diffusion and energy transfer in polydisperse mixtures of particles with different temperatures
}

\author{
Efe Ilker $\odot,{ }^{1,2,3}$ Michele Castellana, ${ }^{1,2}$ and Jean-François Joanny ${ }^{1,2,4}$ \\ ${ }^{1}$ Laboratoire Physico-Chimie Curie, Institut Curie, PSL Research University, CNRS UMR 168, 75005 Paris, France \\ ${ }^{2}$ Sorbonne Universités, UPMC Univ. Paris 06, 75005 Paris, France \\ ${ }^{3}$ Max Planck Institute for the Physics of Complex Systems, 01187 Dresden, Germany \\ ${ }^{4}$ Collège de France, 11 place Marcelin Berthelot, 75005 Paris, France
}

(Received 18 March 2021; accepted 19 May 2021; published 14 June 2021)

\begin{abstract}
Evidence suggests that the transport rate of a passive particle at long time scales is enhanced due to interactions with the surrounding active ones in a size- and composition-dependent manner. Using a system of particles with different temperatures, we probe these effects in dilute solutions and derive long-time friction and self-diffusion coefficients as functions of volume fractions, sizes, and temperatures of particles in $d=2$ and 3 dimensions. Thus, we model excluded-volume interactions for nonequilibrium systems but also extend the scope to short-range soft potentials and compare our results to Brownian-dynamics simulations. Remarkably, we show that both viscosity and energy flux display a nonlinear dependence on size. The simplicity of our formalism allows to discover various interesting scenarios that can be relevant for biological systems and active colloids.
\end{abstract}

DOI: 10.1103/PhysRevResearch.3.023207

\section{INTRODUCTION}

A particle in a solvent is constantly bombarded by the solvent molecules (receiving random kicks at a rate $\sim \tau_{\mathrm{s}}^{-1}$ ) which push the particle, while the particle dissipates the excess energy through dynamical friction also exerted by the solvent at longer time scales. This leads to the well-known Brownian motion [1]. The transport is measured by the mean-square displacement (MSD) of the particle, which is determined by its diffusion constant $D=T / \zeta$ at time periods $t \gg \tau_{\mathrm{s}}$. Here, $T$ is the temperature of the solvent, i.e., the "bath" temperature, and $\zeta$ is the friction coefficient of the particle, which depends on the viscosity of the solvent and the size of the particle. For finite concentrations of interacting particles, at much longer time spans $t \gg \tau_{\mathrm{c}}$ the diffusion constant of a single tagged particle differs from the bare diffusion constant $D$, because the particle experiences numerous collisions with the surrounding particles at a rate $\sim \tau_{\mathrm{c}}^{-1}$. In equilibrium fluids, this effect is a consequence of the effective friction due to the excluded-volume interactions [2] and can only reduce the value of long-time value diffusion constant $D^{\mathrm{s}}$ of the tagged particle, i.e., its self-diffusion coefficient.

By contrast, in an active fluid, a passive tracer particle can gain extra energy through interactions with the surrounding active particles [3-5]. The self-diffusion coefficient is then

Published by the American Physical Society under the terms of the Creative Commons Attribution 4.0 International license. Further distribution of this work must maintain attribution to the author(s) and the published article's title, journal citation, and DOI. Open access publication funded by the Max Planck Society. determined by the interplay between the effective friction discussed above and this energy transfer. In biological systems, crowding, energy transmission, and composition are key factors on the transport of material [6-10], and outof-equilibrium aspects enrich the dynamics. Notably in the cytoplasm, not only the long-time friction scales nonlinearly $[11,12]$, but also the energy flux varies with the size of the probe particle [13]. Disentangling these elements is difficult in general polar active models. Yet, we can attempt to establish simpler models, which keep the relevant features of these systems. In particular, activity brings extra persistence with velocity $v$ to the translational motion of active particles while the direction is randomized at a rotational time scale $\tau_{\mathrm{r}}$. At intermediate time scales $\tau_{\mathrm{c}}>t \gg \tau_{\mathrm{r}}$, this motion appears diffusive with $D \sim v^{2} \tau_{r}$, and can be described by an effective temperature, higher than the thermal one. Similar characteristics can be achieved by phoretic motility in artificial self-propelled particles [14]. While $\tau_{\mathrm{r}}$ is very short for biomolecules, it gains importance for self-propelled particles. At time scales $t<\tau_{c}$, the tagged particle diffusion is only perturbed by hydrodynamic interactions $[15,16]$. A recent study explores how the hydrodynamic field of the microswimmer leads to a ballistic motion of tracer particles at short to intermediate time scales [17]. On the other hand, at long time scales $t \gg \tau_{\mathrm{c}}$, the dynamics converges to diffusive motion where direct collisions dominate and the effect of the hydrodynamic field is decreased in the long-time selfdiffusion coefficient $D^{\mathrm{s}}[18,19]$. This allows for a consistent separation of time scales to implement a multiple-temperature model for studying the long-time transport properties in outof-equilibrium systems.

Various studies using particles with different temperatures explored rich phenomena beyond equilibrium mixtures: 
active/passive phase separation of colloids [20,21], polymeric systems [22-24], activity-mediated interactions [25], interaction-dependent temperatures [26], diffusion of a passive particle weakly coupled to an active field (and vice versa) [27], among others. Recently, we constructed a theory of phase separation for these systems, and showed that the three-body correlations lead to nonreciprocal interactions upon coarse-graining [28] and hence a shift from an effective equilibrium construction. This reflects similar governing principles and peculiarities observed in polar active models $[29,30]$. Moreover, the concept of a second temperature successfully describes other systems, ranging from biology to plasma physics [31-40].

In this paper, we provide a theoretical and numerical analysis of the long-time behavior of transport properties and energy transfer as functions of sizes, temperatures, and concentrations in mixtures of particles with different temperatures. We start from the Langevin dynamics with excluded-volume interactions while neglecting hydrodynamic interactions, and derive the long-time transport coefficients expanded up to first order in concentrations. In Sec. II, we describe the model and highlight the central results of this paper, which we compare with the Brownian dynamics simulations. In Sec. III, we discuss how modeling of excluded-volume interactions in nonequilibrium systems differs from the equilibrium systems. The detailed derivations are included in Supplemental Material [41], which we hope to be useful for further studies in nonequilibrium systems as we extend the scope of microrheology approaches developed in equilibrium systems and simplify the framework.

\section{MODEL AND RESULTS}

We consider the motion of colloidal particles in $d=2,3$ dimensions following overdamped Langevin dynamics

$$
\zeta_{m} \dot{\mathbf{r}}_{m}=-\partial_{\mathbf{r}_{m}} U_{N}+\left(2 T_{m} \zeta_{m}\right)^{1 / 2} \boldsymbol{\xi}_{m}(t) .
$$

The total potential energy $U_{N}=\frac{1}{2} \sum_{i, j} u\left(\left|\mathbf{r}^{(i)}-\mathbf{r}^{(j)}\right|\right)+$ $\sum_{i} u_{\mathrm{v}}\left(\mathbf{r}^{(i)}\right)$ includes both the interactions between particles and an external potential, which confines our system in a volume $V$. The position of particle $i$ is denoted by $\mathbf{r}_{i}$, its friction coefficient by $\zeta_{i}$, its temperature by $T_{i}$ and $\xi_{i}(t)$ is a $d$-dimensional standard Gaussian white noise vector with zero mean and unit variance, i.e., $\left\langle\boldsymbol{\xi}_{i}(t) \cdot \boldsymbol{\xi}_{j}\left(t^{\prime}\right)\right\rangle=d \delta\left(t-t^{\prime}\right) \delta_{i j}$. We consider multiple species of particles. Each particle of species $\alpha$ is in contact with a thermostat at temperature $T_{\alpha}$ and friction coefficient $\zeta_{\alpha}$. In general, the observables of the multiparticle system can be expressed as a series expansion in the particle densities. At first order in concentrations, the correction to isolated particle properties results from the sum of the contributions of all two-particle clusters.

For two particles of species $\alpha$ and $\beta$, respectively, at positions $\mathbf{r}_{1}$ and $\mathbf{r}_{2}$ and interacting through a pairwise potential, which depends only on the distance between the particles, $u_{\alpha \beta} \equiv u_{\alpha \beta}\left(\left|\mathbf{r}_{2}-\mathbf{r}_{1}\right|\right)$, we choose a coordinate system where $\mathbf{r}=\mathbf{r}_{2}-\mathbf{r}_{1}$ is the separation vector and $\mathbf{R}=$ $w \mathbf{r}_{1}+(1-w) \mathbf{r}_{2}$ the center of motion, with $w \equiv D_{\beta} /\left(D_{\alpha}+\right.$ $\left.D_{\beta}\right)=\zeta_{\alpha} T_{\beta} /\left(\zeta_{\alpha} T_{\beta}+\zeta_{\beta} T_{\alpha}\right)$. With this choice, the diffusive motions along the directions $\mathbf{r}$ and $\mathbf{R}$ are statistically independent [20]. As a result, we obtain the two-particle Langevin dynamics in the form:

$$
\begin{aligned}
& \dot{\mathbf{r}}=-\frac{1}{\zeta_{r}^{\alpha \beta}} \partial_{\mathbf{r}} u_{\alpha \beta}(r)+\left(2 D_{r}^{\alpha \beta}\right)^{1 / 2} \xi_{r}(t), \\
& \dot{\mathbf{R}}=-\frac{1}{\zeta_{R}^{\alpha \beta}} \partial_{\mathbf{r}} u_{\alpha \beta}(r)+\left(2 D_{R}^{\alpha \beta}\right)^{1 / 2} \xi_{R}(t)
\end{aligned}
$$

as $\left\langle\boldsymbol{\xi}_{k}(t) \cdot \boldsymbol{\xi}_{l}\left(t^{\prime}\right)\right\rangle=d \delta\left(t-t^{\prime}\right) \delta_{k l}$ and $\left\langle\boldsymbol{\xi}_{k, l}(t)\right\rangle=0$ where $D_{r}^{\alpha \beta}=T_{\alpha \beta} / \zeta_{r} \quad$ and $\quad \zeta_{r}^{\alpha \beta}=\frac{\zeta_{\alpha \beta} \zeta_{\beta}}{\zeta_{\alpha}+\zeta_{\beta}}, \quad T_{\alpha \beta}=\frac{\zeta_{\beta} T_{\alpha}+\zeta_{\alpha} T_{\beta}}{\zeta_{\alpha}+\zeta_{\beta}}, \quad D_{R}^{\alpha \beta}=$ $\frac{T_{\alpha} T_{\beta}}{\zeta_{\alpha} T_{\beta}+\zeta_{\beta} T_{\alpha}}$, and $\zeta_{R}^{\alpha \beta}=\frac{\zeta_{\beta} T_{\alpha}+\zeta_{\alpha} T_{\beta}}{T_{\alpha}-T_{\beta}}$. The advective term in $\dot{\mathbf{R}}$ leads to energy transfer, it only exist for nonequilibrium systems, and it vanishes as $\zeta_{R}^{-1} \rightarrow 0$ for $T_{\alpha}=T_{\beta}$.

We may now proceed to derive long-time dynamical coefficients for a tagged particle of species $\alpha$ that has temperature $T_{\alpha}$, friction coefficient $\zeta_{\alpha}$, and diameter $\sigma_{\alpha}$, which plays a role in excluded-volume interactions. Throughout this paper, we define each secondary particle as type $\beta$, hence $\{\beta\}$ represents a set of different particle types surrounding the tagged particle. Accordingly, for an $\alpha$-type tagged particle, there are $N_{\beta}$ pairs for $\alpha \beta$ interactions (we take $N_{\alpha}-1 \approx N_{\alpha}$ ), and we leverage the statistical equivalence of pair interactions described in Eqs. (2) and (3).

\section{A. Long-time friction coefficient}

At long times, the collisions with the surrounding particles alters the effective friction on the tagged particle. In order to determine the density dependent long-time friction coefficient, we apply a small constant test force $\mathbf{F}$ on a tagged particle of species $\alpha$ at position $\mathbf{r}_{1}$, along the lines of Refs. [42,43]. The test force $\mathbf{F}$ breaks the symmetry of the steady state and creates a nonhomogeneous distribution of the other particles around the tagged one. In return, this alters the force balance and induces an effective mean force $\mathbf{F}_{\text {in }}$ on the tagged particle through the excluded-volume interactions with the surrounding particles. The average velocity follows a linear relation with the total applied forces $\left\langle\mathbf{u}_{\alpha}\right\rangle=$ $\left(\mathbf{F}+\mathbf{F}_{\text {in }}\right) / \zeta_{\alpha}$ from which we can infer the long-time friction coefficient $\zeta_{\alpha}^{\mathrm{L}}$ as we look for $\left\langle\mathbf{u}_{\alpha}\right\rangle=\mathbf{F} / \zeta_{\alpha}^{\mathrm{L}}$. To account for all pair interactions with the tagged particle, we define the concentrations for each species $\{\beta\}$ with $N_{\beta}$ particles of diameter $\sigma_{\beta}$ in a volume $V$ as $c_{\beta}=N_{\beta} / V$, and the volume fractions as $\phi_{\beta}=\frac{\Omega_{d}}{2^{d} d}\left(\sigma_{\beta}\right)^{d} c_{\beta}$, where $\Omega_{d}$ is the solid angle in $d$ dimensions. We obtain [41]

$$
\zeta_{\alpha}^{\mathrm{L}}=\zeta_{\alpha}\left(1+\sum_{\beta} 4 \frac{\zeta_{r}^{\alpha \beta}}{\zeta_{\alpha}}\left(\frac{\sigma_{\alpha \beta}}{\sigma_{\beta}}\right)^{d} \phi_{\beta}\right),
$$

in $d=2,3$ where $\sigma_{\alpha \beta}=\left(\sigma_{\alpha}+\sigma_{\beta}\right) / 2$ is the contact distance. The summation is performed over all particle species, and hence our theory is valid for arbitrary polydisperse compositions with many particle species at linear order in concentrations. Including the size effects on solvent-based friction constants [44], this suggests a weak compositional dependence in $d=2$ in accordance with experimental results [19] and a strong dependence in $d=3$ as we illustrate with simulations below. We should note that the long-time friction coefficients are temperature independent and identical for equilibrium systems of particles in contact with a single 
thermostat and for nonequilibrium systems with the same types of particles having multiple temperatures. This calculation is sufficient to determine the self-diffusion coefficient at linear order in equilibrium systems where $D_{\alpha}^{\mathrm{s}}=$ $D_{\alpha} \zeta_{\alpha} / \zeta_{\alpha}^{\mathrm{L}}$. However, the nonequilibrium counterpart for the self-diffusion coefficient $D_{\alpha}^{\mathrm{s}}$ requires an investigation of the dynamics of the tagged-particle density.

\section{B. Long-time diffusion constant}

The free diffusion constant of an $\alpha$-type particle is given by $D_{\alpha}=T_{\alpha} / \zeta_{\alpha}$ and reflects the behavior at short time scales in the absence of collisions. The long-time diffusion constant is obtained from the asymptotic behavior of the MSD $\left\langle\left(r_{\alpha}(t)-\right.\right.$ $\left.\left.r_{\alpha}(0)\right)^{2}\right\rangle$ of tagged particles as $t \rightarrow \infty$ (averaged over all the $\alpha$ particles from many realizations). We may define

$$
\bar{D}_{\alpha}(t)=\frac{\left\langle\left(r_{\alpha}(t)-r_{\alpha}(0)\right)^{2}\right\rangle}{2 d t} .
$$

By definition, $\bar{D}_{\alpha}\left(0^{+}\right)=D_{\alpha}$ and $\bar{D}_{\alpha}(\infty)=D_{\alpha}^{\mathrm{s}}$.

The self-diffusion constant $D_{\alpha}^{\mathrm{s}}$ is linked to the taggedparticle scattering function $F(\mathbf{k}, t)=\left\langle e^{-i \mathbf{k} \cdot\left(\mathbf{r}_{1}(t)-\mathbf{r}_{1}(0)\right)}\right\rangle$, which is the Fourier transform of the tagged-particle density autocorrelation function. Here, each incident wave is weighted with the steady-state probability distribution of finding the tagged particle at $\mathbf{r}_{1}(0)$ at $t=0$ and the conditional probability of finding the particle at $\mathbf{r}_{1}(t)$ at time $t$ given that it started at $\mathbf{r}_{1}(0)$ (see Supplemental Material [41]). For small values of the Laplace variable $s$ conjugate to $t$, and small wave vector $k$, the Laplace transform of the tagged-particle scattering function can be expanded as $s F(\mathbf{k}, s) \approx 1-D_{\alpha}^{\mathrm{s}} k^{2} / s$. The explicit calculation of $D_{\alpha}^{\mathrm{s}}$ is detailed in Supplemental Material [41]. To first order in concentrations, we obtain

$$
D_{\alpha}^{s}=D_{\alpha}-\sum_{\beta} 4 \frac{\zeta_{r}^{\alpha \beta}}{\zeta_{\alpha}}\left(\frac{T_{\alpha}}{\zeta_{\alpha}}+\frac{T_{\alpha}-T_{\beta}}{\zeta_{\alpha}+\zeta_{\beta}}\right)\left(\frac{\sigma_{\alpha \beta}}{\sigma_{\beta}}\right)^{d} \phi_{\beta}
$$

in $d=2,3$ dimensions [45]. The second term in the sum reflects the nonequilibrium contributions to the self-diffusion constant due to the different temperatures.

To illustrate certain aspects of Eq. (6), we consider a binary system of particles with one single particle of type- $A$ (tracer) surrounded by many type- $B$ particles (crowders), i.e., $\phi_{A} \rightarrow 0$ and $\phi_{B}>0$ while the temperatures differ by an amount $\Delta T=$ $T_{B}-T_{A}$. When $T_{B} \gg T_{A}$ and $\sigma_{A} \gg \sigma_{B}$, the fractional change in root-MSD scales linearly with the size of the tracer, i.e., $\left(\sqrt{D_{A}^{\mathrm{s}}}-\sqrt{D_{A}}\right) / \sqrt{D_{A}} \sim \sigma_{A}$, remarkably similar to the observations on tracer diffusion in bacterial cytoplasm where the metabolic activity is tuned by energy depletion procedures [13]. For equal-sized $A$ and $B$ particles with particle size $\sigma_{A}=$ $\sigma_{B}$ and $\zeta_{A}=\zeta_{B}=\zeta$, the self-diffusion constant of particle $A$ becomes $D_{A}^{\mathrm{s}}=D_{A}\left(1-2 \phi_{B}\right)+(\Delta T / \zeta) \phi_{B}$. For equilibrium systems, $\Delta T=0$, and the self-diffusion constant recovers the known value $D_{A}^{\mathrm{s}}=D_{A}\left(1-2 \phi_{B}\right)[18,46]$. For nonequilibrium systems, as $\Delta T \neq 0$, we investigate two opposite limits: (i) When $T_{A} \gg T_{B}, D_{A}^{\mathrm{s}} \approx D_{A}\left(1-3 \phi_{B}\right)$ increasing the slowing down of the hot particle by the cold crowders. This effect is also observed with active Brownian particles for which the propulsion speed is more damped [47] when the surrounding bath is composed of less active particles [48]. (ii) When
$T_{A} \ll T_{B}, D_{A}^{\mathrm{s}} \approx D_{B} \phi_{B}$ such that the diffusion of cold particles is mainly driven by hot crowders.

\section{Modified Einstein relation and effective temperatures}

We can define an effective temperature $T_{\alpha}^{\text {eff }}$ for the tagged particle by imposing an Einstein relation $D_{\alpha}^{\mathrm{s}}=T_{\alpha}^{\mathrm{eff}} / \zeta_{\alpha}^{\mathrm{L}}$ (see Supplemental Material [41]), and we find

$$
T_{\alpha}^{\mathrm{eff}}=T_{\alpha}-\sum_{\beta} 4 \frac{\zeta_{\alpha} \zeta_{\beta}}{\left(\zeta_{\alpha}+\zeta_{\beta}\right)^{2}}\left(T_{\alpha}-T_{\beta}\right)\left(\frac{\sigma_{\alpha \beta}}{\sigma_{\beta}}\right)^{d} \phi_{\beta} .
$$

Thus, this measure corresponds only to the translational motion and the difference between the effective temperature and the real thermostat temperature reflects the energy exchange between hot and cold particles. For binary mixtures of equal-sized hard spheres, this simplifies to $T_{A}^{\text {eff }}=T_{A}+$ $\Delta T \phi_{B}$. Since the interaction potential is conservative the total energy conservation imposes that $[22,24]$

$$
\sum_{\alpha} T_{\alpha} c_{\alpha}=\sum_{\alpha} T_{\alpha}^{\mathrm{eff}} c_{\alpha}
$$

which naturally follows from Eq. (7).

\section{Brownian dynamics simulations and results in $\mathbf{3}$ dimensions}

To test our predictions, we perform Brownian dynamics simulations of Eq. (1) with a soft repulsive pairwise potential, $v_{\alpha \beta}(r)=\frac{k}{2}\left(\sigma_{\alpha \beta}-r\right)^{2}$ for $r<\sigma_{\alpha \beta}$ and $v_{\alpha \beta}(r)=0$ otherwise. For a short-range potential with sufficiently narrow cut-off, an effective hard-core interaction diameter can be defined in $d=2,3$, and it reads

$$
\sigma_{\alpha \beta}^{\prime}=\left(\frac{d B_{\alpha \beta}}{\Omega_{d}}\right)^{1 / d},
$$

where $B_{\alpha \beta}=\Omega_{d} \int\left(1-e^{-v_{\alpha \beta}(r) / T_{\alpha \beta}}\right) r^{d-1} d r$ is the second virial coefficient [20,28], which recovers $\sigma_{\alpha \beta}^{\prime}=\left(\sigma_{\alpha}+\sigma_{\beta}\right) / 2$ for hard spheres. This transformation allows for an equivalent hard-sphere description with scaled contact distances [49-52] using which we can compare the simulation results with our analytical formulas from Eqs. (4), (6), and (7) by replacing $\sigma_{\alpha \beta}$ with $\sigma_{\alpha \beta}^{\prime}$ (see Supplemental Material for details [41]).

In Fig. 1(a), we show $\bar{D}_{\alpha}(t)$ [Eq. (5)] obtained from simulations and the fitting curve from which we determine the $D_{\alpha}^{\mathrm{s}}$ values for two examples a tagged hot particle in a cold particle bath (orange) and a tagged cold particle in a hot particle bath (blue). We extract $D_{\alpha}^{\mathrm{s}}$ by extrapolating Eq. (5) with a stretched exponential, i.e., $\bar{D}_{\alpha}(t)=D_{\alpha}+\left(D_{\alpha}^{\mathrm{s}}-D_{\alpha}\right)\left(1-e^{-(t / \tau)^{\gamma}}\right)$ and typically $\gamma \approx 1 / 2$ as previously employed in hard-sphere simulations [53]. The details on the simulations and their analysis are given in Sec.V of the Supplemental Material [41]. The size dependence enters in Eqs. (4), (6), and (7), both through the contact distance $\sigma_{\alpha \beta}$ and the friction coefficient, which follow Stokes' law $\zeta_{\alpha} \sim \sigma_{\alpha}$. We first consider the behavior of a tagged particle $A$ in a bath of crowders $B$. In all examples, we set $\sigma_{B}=\sigma$, total volume $V=5 \times 10^{4} \pi \sigma^{3} / 6, \kappa \equiv$ $k \sigma^{2} / T_{\max }=10^{2}$, where $T_{\max }=\max \left(T_{A}, T_{B}\right)$. In Fig. 1(b), we observe the size and concentration dependence of the long-time friction coefficient. The increase with radius is stronger than the Stokes' law as the concentration of crowders increases. Equation (4) suggests that $\zeta_{A}^{\mathrm{L}} \sim \sigma_{A}\left[1+\epsilon\left(\phi_{B}\right) \sigma_{A B}^{2}\right]$ 


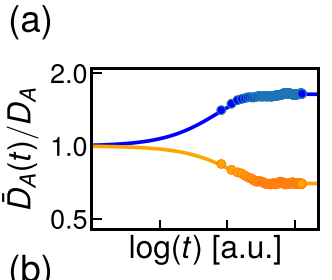

(b)

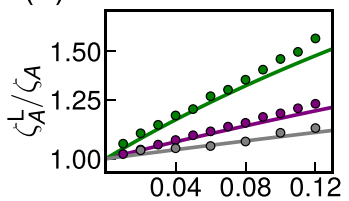

(c)
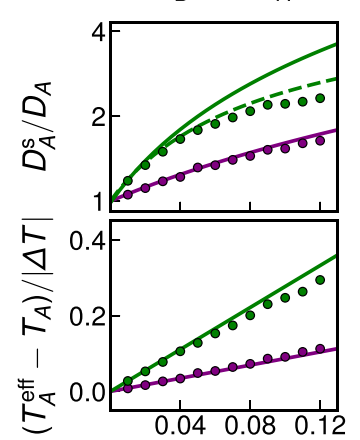

(d)
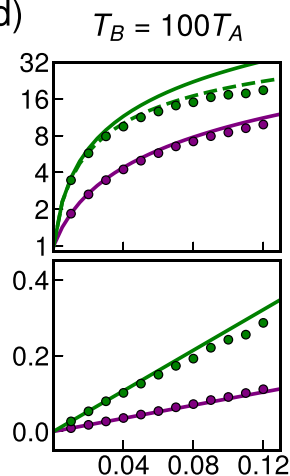

(e)

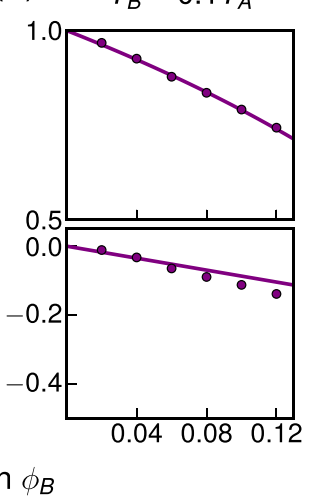

(f)

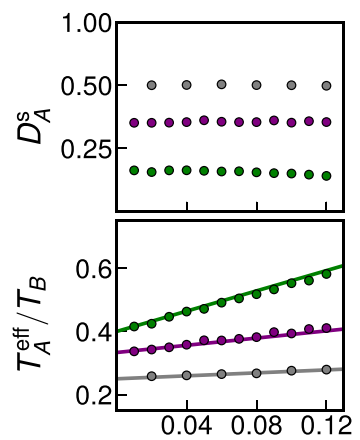

Crowder volume fraction $\phi_{B}$

FIG. 1. Tagged particle $A$ in a bath of crowders $B$ in $d=3$. (a) $\bar{D}_{A}(t)$ from simulations [Eq. (5), shown in log scale] as a function of time interval $\log (t)$ for two examples; cold particle in hot bath (blue), hot particle in cold bath (orange). In (b)-(f), the color code depicts size ratios $\sigma_{A} / \sigma_{B}=0.5,1,2$ in gray, purple, and green, respectively, and dots are obtained from simulations, solid lines represent theoretical results for friction coefficient, self-diffusion coefficient, and effective temperature shift, respectively, from Eqs. (4), (6), and (7) with scaled contact distances $\sigma_{\alpha \beta} \rightarrow \sigma_{\alpha \beta}^{\prime}$. In (b), the long-time friction coefficient increases with crowder volume fraction $\phi_{B}$ and displays a behavior beyond Stokes' law as $\zeta_{A}^{\mathrm{L}} \sim \sigma_{A}\left[1+\epsilon\left(\phi_{B}\right) \sigma_{A B}{ }^{2}\right]$ where $\epsilon\left(\phi_{B}\right)=4 \phi_{B} / \sigma_{B}^{2}$. Panels (c)-(e) show how both self-diffusion (shown in log scale) and temperature vary with sizes, temperatures, and volume fraction of crowders. A slight discrepancy between the theory, Eq. (6), and simulations for self-diffusion arises for larger tracer particles for which Eq. (10) (dashed lines) show better success. In (f), we show three illustrative cases where the energy transfer balances frictional forces for temperature ratios satisfying Eq. (11).

where $\epsilon\left(\phi_{B}\right)=4 \phi_{B} / \sigma_{B}^{2}$. This nonlinear scaling of the friction constant with increasing tracer size is observed in cytoplasmic transport and polymeric systems [11]. Although the interactions in those systems are not limited to steric interactions but also include hydrophobic and electrostatic interactions, one can use our formalism extended to narrow-range soft potentials, which defines an effective contact distance $\sigma_{\alpha \beta}^{\prime}$. In Figs. 1(c)-1(e), we show the self-diffusion constant and energy transfer, respectively, for $T_{B}=10 T_{A}, T_{B}=100 T_{A}$, $T_{B}=0.1 T_{A}$. Our theory fits particularly well the simulations for the effective temperatures, whereas we observe slight discrepancy in self-diffusion constant as the size ratio $\sigma_{A} / \sigma_{B}$ increases. To propose a correction, we attempted to write

$$
D_{\alpha}^{s \dagger}=\frac{T_{\alpha}^{\mathrm{eff}}}{\zeta_{\alpha}^{\mathrm{L}}},
$$

using $T_{\alpha}^{\text {eff }}$ from Eq. (7), and $\zeta_{\alpha}^{\mathrm{L}}$ from Eq. (4). The outcome is shown by dashed lines and and slightly improves the agreement with the simulation results. It obviously recovers the rigorously-derived Eq. (6) at linear order, while it is an uncontrolled expansion at higher orders.

An interesting limit is that where energy transfer balances the friction from the same type of surrounding particles. This happens when

$$
\frac{T_{A}}{T_{B}}=\frac{1}{2+\sigma_{B} / \sigma_{A}},
$$

then $D_{A}^{\mathrm{s}}=D_{A}$ becomes insensitive to $\phi_{B}$, see Fig. 1(f). Thus, the friction of the $B$ bath vanishes and the crowder particles appear completely transparent to the tracer particle. The motion remains diffusive (only solvent-based) at all time scales (but strictly $t \gg \tau_{\mathrm{r}}$ for systems with active swimmers) as the only process is collisions with nonviscous crowders.

Next, we study binary mixtures of equal-sized particles at finite concentrations, e.g., $\phi_{A}=\phi_{B}=0.025$ and $T_{B} / T_{A}>1$. We also investigate the dependence on potential stiffness and the self-diffusion coefficients converge to the hard-sphere values with increasing $\kappa$ as $\lim _{\kappa \rightarrow \infty} \sigma_{\alpha \beta}^{\prime}=\sigma_{\alpha \beta}$. Upon increasing the temperature ratio, the fractional increment in diffusion coefficient of cold particles is significant and follows a linear trend. On the other hand, the fractional loss for hot particles is less sensitive to $T_{B} / T_{A}$ and converges to $D_{B}^{\mathrm{s}} / D_{B}=1-3 \phi_{A}-$ $2 \phi_{B}$ as $T_{B} \gg T_{A}$ (and $\kappa \rightarrow \infty$ ) predicted by our theory. We also verified the energy conservation (8) for the same system [Fig. 2(b)]. This holds irrespective of the potential stiffness and the energy transfer in this example more strictly suggests $T_{A}^{\text {eff }}+T_{B}^{\text {eff }}=T_{A}+T_{B}$ since $c_{A}=c_{B}$.

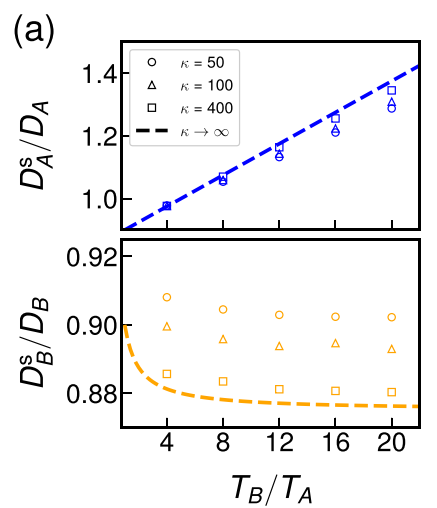

(b)

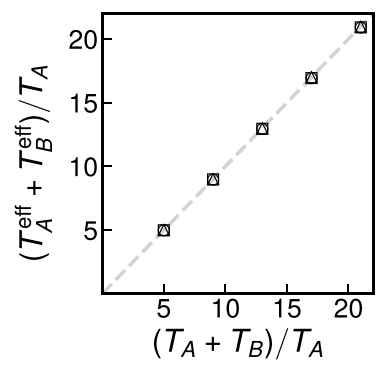

FIG. 2. Mixtures, $\kappa$ dependency, and energy conservation. In (a), we show the self-diffusion coefficients in free-diffusion coefficient units for equal-sized cold (top) and hot (bottom) particle species in a mixture with $\phi_{A}=\phi_{B}=0.025$ for varying values of potential stiffness $\kappa=50,100,400$, respectively, shown as circles, triangles, and squares. As $\kappa \rightarrow \infty$, the self-diffusion coefficients converge to the hard-sphere values (dashed lines). In (b), we display the energy conservation Eq. (8) resulting the condition $T_{A}^{\text {eff }}+T_{B}^{\text {eff }}=T_{A}+T_{B}$ for $c_{A}=c_{B}$, which is conserved irrespective of the potential stiffness. 


\section{NATURE OF EXCLUDED-VOLUME INTERACTIONS}

On a final note, we stress a crucial distinction on how to model excluded-volume interactions in nonequilibrium systems. For real-world systems, the excluded-volume refers to repulsive steric interactions. In equilibrium systems, these can be treated as a boundary condition for the moving particle in which interparticle distances $r<\sigma_{\alpha \beta}$ are not allowed. This would produce the same statistics for more realistic potentials with nearly hard-sphere descriptions (e.g., Lennard-Jones potential at high temperatures) while both simplifying analytical approaches and allowing for Monte Carlo methods. However, in such description, the diffusion of a moving particle is always constrained by the existence of the surrounding particles. Thus, even for the colder particle, we would expect no increase in diffusivity, such that $D_{\alpha}^{\mathrm{s}} \leqslant D_{\alpha}$ for all temperature ratios. On the other hand, this would no longer be an obligation for repulsive interactions, and the cold particle can gain energy through collisions. Conventional Monte Carlo methods would fail to mimic this effect as jump rates are only constrained - and not enhanced-by the existence of another particle. Yet, a proper description can be recovered in lattice models by adding a simple collision rule as demonstrated in Ref. [54].

Here, our derivation conserves the analytical properties of pair distribution functions, and enables us to approach the hard-sphere limit through a smooth function (and vice versa towards soft potentials). We are therefore able to capture nonequilibrium aspects while keeping the simplicity of a hardsphere description.

\section{CONCLUSIONS}

In this paper, we have identified the effect of energy flux and friction on the long-time self-diffusion constant of colloidal particles. We intended to study nonequilibrium mix- tures, but Eq. (6) also provides an explicit formula that can be used in equilibrium systems of polydisperse solutions at $T_{\alpha}=T_{\beta}=T$ (more generally with $\sigma_{\alpha \beta} \rightarrow \sigma_{\alpha \beta}^{\prime}$ ). We left out the effect of hydrodynamic interactions in our current analysis, where we focused on the long-time dynamics. However, hydrodynamic interactions could be included in our framework. Yet this would be a more ambitious undertaking, because also there is no consensus even for equilibrium systems on the significance of the contribution of hydrodynamic interactions to the long-time value of the self-diffusion coefficient $D_{\alpha}^{s}$,compared to the value only due to direct collisions [16,18,55-57]. It would be interesting to investigate these effects on tagged particle dynamics at different time windows in a study complemented by experimental results.

We hope that our theory will also shed light on experimental studies of active colloids, microswimmers, and single-molecule dynamics in biological mixtures such as the cytoplasm. Notably, the theoretical predictions on the size scalings of diffusion enhancement and long-time friction at dilute concentrations of crowders show close resemblance with earlier experimental results on the cytoplasmic transport. Even though we set our theory in the mixing regime, it can be adapted to complex fluids that have spatial heterogeneities by introducing local values of the volume fractions. This would require further study and we leave this to a future work.

\section{ACKNOWLEDGMENTS}

We thank A. S. Vishen, V. Démery, and M. Delarue for interesting discussions. We acknowledge support from the Labex CelTisPhyBio (Grants No. ANR-11-LABX-0038, No. ANR-10-IDEX-0001-02) and from Agence Nationale de la Recherche (ANR), Grant No. ANR-17-CE11-0004.
[1] A. Einstein, On the motion of small particles suspended in liquids at rest required by the molecular-kinetic theory of heat, Ann. Phys. 17, 549 (1905).

[2] J. A. Leegwater and G. Szamel, Dynamical properties of hardsphere suspensions, Phys. Rev. A 46, 4999 (1992).

[3] X.-L. Wu and A. Libchaber, Particle Diffusion in a Quasi-TwoDimensional Bacterial Bath, Phys. Rev. Lett. 84, 3017 (2000).

[4] K. C. Leptos, J. S. Guasto, J. P. Gollub, A. I. Pesci, and R. E. Goldstein, Dynamics of Enhanced Tracer Diffusion in Suspensions of Swimming Eukaryotic Microorganisms, Phys. Rev. Lett. 103, 198103 (2009).

[5] G. Mino, T. E. Mallouk, T. Darnige, M. Hoyos, J. Dauchet, J. Dunstan, R. Soto, Y. Wang, A. Rousselet, and E. Clement, Enhanced Diffusion Due to Active Swimmers at a Solid Surface, Phys. Rev. Lett. 106, 048102 (2011).

[6] A. P. Minton, The influence of macromolecular crowding and macromolecular confinement on biochemical reactions in physiological media, J. Biol. Chem. 276, 10577 (2001).

[7] R. J. Ellis, Macromolecular crowding: Obvious but underappreciated, Trends Biochem. Sci. 26, 597 (2001).
[8] M. Delarue, G. Brittingham, S. Pfeffer, I. Surovtsev, S. Pinglay, K. Kennedy, M. Schaffer, J. Gutierrez, D. Sang, G. Poterewicz et al., mTORC1 controls phase separation and the biophysical properties of the cytoplasm by tuning crowding, Cell 174, 338 (2018).

[9] M. A. Vibhute, M. H. Schaap, R. J. Maas, F. H. Nelissen, E. Spruijt, H. A. Heus, M. M. Hansen, and W. T. Huck, Transcription and translation in cytomimetic protocells perform most efficiently at distinct macromolecular crowding conditions, ACS Synth. Biol. 9, 2797 (2020).

[10] A. Movilla Miangolarra, A. Duperray-Susini, M. Coppey, and M. Castellana, Two time scales control the creation of large protein aggregates in cells, arXiv:2007.16015.

[11] T. Kalwarczyk, N. Ziebacz, A. Bielejewska, E. Zaboklicka, K. Koynov, J. Szymanski, A. Wilk, A. Patkowski, J. Gapinski, H.-J. Butt et al., Comparative analysis of viscosity of complex liquids and cytoplasm of mammalian cells at the nanoscale, Nano Lett. 11, 2157 (2011).

[12] F. Etoc, E. Balloul, C. Vicario, D. Normanno, D. Liße, A. Sittner, J. Piehler, M. Dahan, and M. Coppey, Non-specific 
interactions govern cytosolic diffusion of nanosized objects in mammalian cells, Nat. Mater. 17, 740 (2018).

[13] B. R. Parry, I. V. Surovtsev, M. T. Cabeen, C. S. O'Hern, E. R. Dufresne, and C. Jacobs-Wagner, The bacterial cytoplasm has glass-like properties and is fluidized by metabolic activity, Cell 156, 183 (2014).

[14] P. Illien, R. Golestanian, and A. Sen, 'Fuelled' motion: Phoretic motility and collective behaviour of active colloids, Chem. Soc. Rev. 46, 5508 (2017).

[15] Z. Cheng, J. Zhu, P. M. Chaikin, S.-E. Phan, and W. B. Russel, Nature of the divergence in low shear viscosity of colloidal hard-sphere dispersions, Phys. Rev. E 65, 041405 (2002).

[16] A. Van Blaaderen, J. Peetermans, G. Maret, and J. Dhont, Long-time self-diffusion of spherical colloidal particles measured with fluorescence recovery after photobleaching, J. Chem. Phys. 96, 4591 (1992).

[17] K. Kanazawa, T. G. Sano, A. Cairoli, and A. Baule, Loopy Lévy flights enhance tracer diffusion in active suspensions, Nature (London) 579, 364 (2020).

[18] S. Hanna, W. Hess, and R. Klein, Self-diffusion of spherical Brownian particles with hard-core interaction, Physica A 111, 181 (1982).

[19] A. L. Thorneywork, D. G. A. L. Aarts, J. Horbach, and R. P. A. Dullens, Self-diffusion in two-dimensional binary colloidal hard-sphere fluids, Phys. Rev. E 95, 012614 (2017).

[20] A. Y. Grosberg and J.-F. Joanny, Nonequilibrium statistical mechanics of mixtures of particles in contact with different thermostats, Phys. Rev. E 92, 032118 (2015).

[21] S. N. Weber, C. A. Weber, and E. Frey, Binary Mixtures of Particles with Different Diffusivities Demix, Phys. Rev. Lett. 116, 058301 (2016).

[22] J. Smrek and K. Kremer, Small Activity Differences Drive Phase Separation in Active-Passive Polymer Mixtures, Phys. Rev. Lett. 118, 098002 (2017).

[23] J. Smrek and K. Kremer, Interfacial properties of active-passive polymer mixtures, Entropy 20, 520 (2018).

[24] I. Chubak, C. N. Likos, K. Kremer, and J. Smrek, Emergence of active topological glass through directed chain dynamics and nonequilibrium phase segregation, Phys. Rev. Research 2, 043249 (2020).

[25] H. Tanaka, A. A. Lee, and M. P. Brenner, Hot particles attract in a cold bath, Phys. Rev. Fluids 2, 043103 (2017).

[26] M. Wang and A. Y. Grosberg, Three-body problem for langevin dynamics with different temperatures, Phys. Rev. E 101, 032131 (2020).

[27] V. Démery and D. S. Dean, Perturbative path-integral study of active-and passive-tracer diffusion in fluctuating fields, Phys. Rev. E 84, 011148 (2011).

[28] E. Ilker and J.-F. Joanny, Phase separation and nucleation in mixtures of particles with different temperatures, Phys. Rev. Research 2, 023200 (2020).

[29] A. P. Solon, J. Stenhammar, M. E. Cates, Y. Kafri, and J. Tailleur, Generalized thermodynamics of motility-induced phase separation: Phase equilibria, laplace pressure, and change of ensembles, New J. Phys. 20, 075001 (2018).

[30] E. Tjhung, C. Nardini, and M. E. Cates, Cluster Phases and Bubbly Phase Separation in Active Fluids: Reversal of the Ostwald Process, Phys. Rev. X 8, 031080 (2018).
[31] V. S. Pande, A. Y. Grosberg, and T. Tanaka, Heteropolymer freezing and design: Towards physical models of protein folding, Rev. Mod. Phys. 72, 259 (2000).

[32] R. Exartier and L. Peliti, A simple system with two temperatures, Phys. Lett. A 261, 94 (1999).

[33] L. F. Cugliandolo, The effective temperature, J. Phys. A 44, 483001 (2011).

[34] I. Abdoli, E. Kalz, H. D. Vuijk, R. Wittmann, J.-U. Sommer, J. M. Brader, and A. Sharma, Correlations in multithermostat Brownian systems with lorentz force, New J. Phys. 22, 093057 (2020).

[35] R. R. Netz, Approach to equilibrium and nonequilibrium stationary distributions of interacting many-particle systems that are coupled to different heat baths, Phys. Rev. E 101, 022120 (2020).

[36] A. Crisanti, A. Puglisi, and D. Villamaina, Nonequilibrium and information: The role of cross correlations, Phys. Rev. E 85, 061127 (2012).

[37] A. Chertovich, E. Govorun, V. Ivanov, P. Khalatur, and A. Khokhlov, Conformation-dependent sequence design: Evolutionary approach, Eur. Phys. J. E 13, 15 (2004).

[38] L. Pitaevskii and E. Lifshitz, Physical Kinetics (ButterworthHeinemann, Oxford, 2012), Vol. 10.

[39] K. M. Ferriere, The interstellar environment of our galaxy, Rev. Mod. Phys. 73, 1031 (2001).

[40] M. G. Wolfire, C. F. McKee, D. Hollenbach, and A. Tielens, Neutral atomic phases of the interstellar medium in the galaxy, Astrophys. J. 587, 278 (2003).

[41] See Supplemental Material at http://link.aps.org/supplemental/ 10.1103/PhysRevResearch.3.023207 for detailed derivations of the long-time transport coefficients given in Eqs. (4), (6) and (7), hard-sphere description of soft narrow-range potentials, and details of the Brownian dynamics simulations.

[42] H. Lekkerkerker and J. Dhont, On the calculation of the selfdiffusion coefficient of interacting Brownian particles, J. Chem. Phys. 80, 5790 (1984).

[43] C. Van Den Broeck, An exact result for the incoherent scattering function in dilute suspensions, J. Chem. Phys. 82, 4248 (1985).

[44] We use Stokes' values for the friction coefficients while we still neglect hydrodynamic interactions that arises from the flow field.

[45] The value of the long-time diffusion constant is negative infinity in $d=1$ for hard spheres [46].

[46] B. J. Ackerson and L. Fleishman, Correlations for dilute hard core suspensions, J. Chem. Phys. 76, 2675 (1982).

[47] J. Stenhammar, R. Wittkowski, D. Marenduzzo, and M. E. Cates, Activity-Induced Phase Separation and Self-Assembly in Mixtures of Active and Passive Particles, Phys. Rev. Lett. 114, 018301 (2015).

[48] In fact, a naive mapping from this work [47], would suggest $D_{A}^{\mathrm{s}} \approx D_{A}\left(1-\beta \phi_{B}\right)$ with $\beta=2.42$. The reduction is larger than the equilibrium result, i.e., $\beta>2$ but lower than the hard-sphere prediction as $\beta<3$ since the pairwise potential is a soft one [see Eq. (9)].

[49] J. A. Barker and D. Henderson, Perturbation theory and equation of state for fluids. II. A successful theory of liquids, J. Chem. Phys. 47, 4714 (1967). 
[50] J.-P. Hansen and I. R. McDonald, Theory of Simple Liquids (Cambridge University Press, Cambridge, 1995), Vol. 1.

[51] J. Rowlinson, The statistical mechanics of systems with steep intermolecular potentials, Mol. Phys. 8, 107 (1964).

[52] H. C. Andersen, J. D. Weeks, and D. Chandler, Relationship between the hard-sphere fluid and fluids with realistic repulsive forces, Phys. Rev. A 4, 1597 (1971).

[53] B. Cichocki and K. Hinsen, Dynamic computer simulation of concentrated hard sphere suspensions: I. Simulation technique and mean square displacement data, Physica A 166, 473 (1990).
[54] L. Abbaspour and S. Klumpp, Enhanced diffusion of a tracer particle in a lattice model of a crowded active system, Phys. Rev. E 103, 052601 (2021).

[55] G. Batchelor, Diffusion in a dilute polydisperse system of interacting spheres, J. Fluid Mech. 131, 155 (1983).

[56] B. Cichocki and B. Felderhof, Long-time self-diffusion coefficient and zero-frequency viscosity of dilute suspensions of spherical Brownian particles, J. Chem. Phys. 89, 3705 (1988).

[57] K. Zahn, J. M. Méndez-Alcaraz, and G. Maret, Hydrodynamic Interactions May Enhance the Self-Diffusion of Colloidal Particles, Phys. Rev. Lett. 79, 175 (1997). 